\title{
The hyperstability of AQ-Jensen functional equation on 2-divisible abelian group and inner product spaces
}

Iz-iddine EL-Fassi* and Samir Kabbaj

Abstract. In this paper, we prove the hyperstability of the following mixed additive-quadratic-Jensen functional equation

$$
2 f\left(\frac{x+y}{2}\right)+f\left(\frac{x-y}{2}\right)+f\left(\frac{y-x}{2}\right)=f(x)+f(y)
$$

in the class of functions from an 2-divisible abelian group $G$ into a Banach space.

AMS Subject Classification (2000). Primary 39B82, 39B52 Secondary 47H14, 47H10.

Keywords. Hyperstability, additive-quadratic-Jensen Functional Equation, fixed point theorem.

\section{Introduction and Preliminaries}

The study of stability problems for functional equations is related to a question of Ulam [29] concerning the stability of group homomorphisms and affirmatively answered for Banach spaces by Hyers [16]. The result of Hyers was generalized by Aoki [2] for approximate additive mappings. In 1978, Th.

${ }^{*}$ Corresponding author 
M. Rassias [27] generalized the result of Hyers by considering the stability problem for unbounded Cauchy differences. This phenomenon of stability introduced by Th. M. Rassias [27] is called the Hyers-Ulam-Rassias stability.

Theorem 1.1 ([27, Th. M. Rassias]). Let $f: E_{1} \rightarrow E_{2}$ be a mapping from a real normed vector space $E_{1}$ into a Banach space $E_{2}$ satisfying the inequality

$$
\|f(x+y)-f(x)-f(y)\| \leq \theta\left(\|x\|^{p}+\|y\|^{p}\right),
$$

for all $x, y \in E_{1}$, where $\theta$ and $p$ are constants with $\theta>0$ and $p<1$. Then there exists a unique additive mapping $T: E_{1} \rightarrow E_{2}$ such that

$$
\|f(x)-T(x)\| \leq \frac{2 \theta}{2-2^{p}}\|x\|^{p}
$$

for all $x \in E_{1}$. If $p<0$ then inequality (1.1) holds for all $x, y \neq 0$, and (1.2) for $x \neq 0$. Also, if the function $t \rightarrow f(t x)$ from $\mathbb{R}$ into $E_{2}$ is continuous for each fixed $x \in E_{1}$, then $T$ is linear.

In 1994, a generalization of Rassias' theorem was obtained by Găvruta [13], who replaced $\theta\left(\|x\|^{p}+\|y\|^{p}\right)$ by a general control function $\varphi(x, y)$.

The stability problems of several functional equations have been extensively investigated by a number of authors and there are many interesting results concerning this problem (see [3,11,14,17-22, 24, 25, 28]).

Recently, interesting results concerning additive-quadratic-Jensen type functional equation (briefly, AQ-Jensen functional equation)

$$
2 f\left(\frac{x+y}{2}\right)+f\left(\frac{x-y}{2}\right)+f\left(\frac{y-x}{2}\right)=f(x)+f(y),
$$

have been obtained in [1] and [30].

We say a functional equation $\mathfrak{D}$ is hyperstable if any function $f$ satisfying the equation $\mathfrak{D}$ approximately is a true solution of $\mathfrak{D}$. The term hyperstability was used for first time probably in [23]. However, it seems that the first hyperstability result was published in [5] and concerned the ring homomorphisms. The hyperstability results for Cauchy equation were investigated by Brzdek in $[7,8,10]$. Gselmann in [15] studied the hyperstability of the parametric fundamental equation of information. In [4] Bahyrycz and Piszczek provided the hyperstability of the Jensen functional equation. In [12] EL-Fassi and Kabbaj studied the hyperstability of a Cauchy-Jensen type functional equation in Banach spaces. 
Vol. LIII (2015) The hyperstability of a mixed additive-quadratic-Jensen... 6

Throughout this paper, we will denote the set of natural numbers by $\mathbb{N}$, the set of integers by $\mathbb{Z}$ and the set of real numbers by $\mathbb{R}$. Let $\mathbb{N}^{*}$ be the set of positive integers. We note that $\mathbb{N}_{m_{0}}$ (with $m_{0} \in \mathbb{N}^{*}$ ) the set of all integers greater than or equal to $m_{0}$. Let $\mathbb{R}_{+}:=[0, \infty)$ be the set of nonnegative real numbers and $\mathbb{R}_{*+}:=(0, \infty)$ the set of positive real numbers and $Y^{X}$ denotes the family of all functions mapping from a nonempty set $X$ into a nonempty set $Y$.

J. Brzdek and K. Ciepliński [9] introduced the following definition, which describes the main ideas of such a hyperstability notion for equations in several variables.

Definition 1.1. Let $X$ be a nonempty set, $(Y, d)$ be a metric space, $\varepsilon: X^{n} \rightarrow$ $\mathbb{R}_{+}$(with $n \in \mathbb{N}^{*}$ ) be an arbitrary function, and let $\mathcal{F}_{1}, \mathcal{F}_{2}$ be two operators mapping from a nonempty set $\mathcal{D} \subset Y^{X}$ into $Y^{X^{n}}$. We say that the operator equation

$$
\mathcal{F}_{1} \varphi\left(x_{1}, \ldots, x_{n}\right)=\mathcal{F}_{2} \varphi\left(x_{1}, \ldots, x_{n}\right), \quad\left(x_{1}, \ldots, x_{n} \in X\right)
$$

is $\varepsilon$-hyperstable provided that every $\varphi_{0} \in \mathcal{D}$ which satisfies

$$
d\left(\mathcal{F}_{1} \varphi_{0}\left(x_{1}, \ldots, x_{n}\right), \mathcal{F}_{2} \varphi_{0}\left(x_{1}, \ldots, x_{n}\right)\right) \leq \varepsilon\left(x_{1}, \ldots, x_{n}\right), \quad\left(x_{1}, \ldots, x_{n} \in X\right)
$$

fulfills equation (1.4) on $X$.

In this article, we introduce the following definition, which describes the main ideas of the concept of hyperstability for equations in several variables.

Definition 1.2. Let $X$ be a nonempty set, $(Y, d)$ be a metric space, $\Sigma \subset \mathbb{R}_{*+}^{X^{n}}$ be a nonempty subset and $\mathcal{F}_{1}, \mathcal{F}_{2}$ be operators mapping from a nonempty set $\mathcal{D} \subset Y^{X}$ into $Y^{X^{n}}$ We say that the operator equation

$$
\mathcal{F}_{1} \varphi\left(x_{1}, \ldots, x_{n}\right)=\mathcal{F}_{2} \varphi\left(x_{1}, \ldots, x_{n}\right), \quad\left(x_{1}, \ldots, x_{n} \in X\right)
$$

is $\Sigma$-hyperstable for the pair $(X, Y)$ provided for any $\varepsilon \in \Sigma$ and $\varphi_{0} \in \mathcal{D}$ satisfies the inequality

$$
d\left(\mathcal{F}_{1} \varphi_{0}\left(x_{1}, \ldots, x_{n}\right), \mathcal{F}_{2} \varphi_{0}\left(x_{1}, \ldots, x_{n}\right)\right) \leq \varepsilon\left(x_{1}, \ldots, x_{n}\right), \quad\left(x_{1}, \ldots, x_{n} \in X\right)
$$

fulfills equation (1.5) on $X$.

A function $H: \mathbb{R}_{+}^{2} \rightarrow \mathbb{R}_{+}$is called homogeneous of degree a real number $p$ if it satisfies $H(t u, t v)=t^{p} H(u, v)$ for all $t \in \mathbb{R}_{*+}$ and $u, v \in \mathbb{R}_{+}$. In the sequel, we assume that $G=(G,+)$ is an 2-divisible abelian group, $E$ is an arbitrary real Banach space, $H: \mathbb{R}_{+}^{2} \rightarrow \mathbb{R}_{+}$is a symmetric homogeneous 
function of degree $p<0$ for which there exists a positive integer $m_{0}$ such that

$$
\inf \left\{\varepsilon(x, m x): m \in \mathbb{N}_{m_{0}}\right\}=0
$$

for all $x \in G$ and $\gamma: G \rightarrow \mathbb{R}_{*+}$ is a function satisfying

(C1) $\gamma\left(\frac{k x}{2}\right)=\frac{|k|}{2} \gamma(x)$ for all $x \in G$ and $k \in \mathbb{Z} \backslash\{0\}$,

(C2) $\gamma(x+y) \leq \gamma(x)+\gamma(y)$ for all $x, y \in G$.

We will denote by $\Sigma$ the set of all functions $\varepsilon: G^{2} \rightarrow \mathbb{R}_{+}$for which there exists a constant $c \in \mathbb{R}_{+}$such that

$$
\varepsilon(x, y)=c H(\gamma(x), \gamma(y)) \quad x, y \in G .
$$

By the conditions (C1) and (C2), we notice that:

$$
\varepsilon\left(\frac{k x}{2}, \frac{k y}{2}\right)=\left|\frac{k}{2}\right|^{p} \varepsilon(x, y)
$$

for all $x, y \in G$ and $k \in \mathbb{Z} \backslash\{0\}$.

In this paper, we present the hyperstability results for the mixed additivequadratic-Jensen type functional equation (1.3) in the class of functions from an 2-divisible commutative group $(G,+)$ into a Banach space $E$.

The method of the proof of the main results is motivated by an idea used in $[7-10,26]$. It is based on a fixed point theorem for functional spaces obtained by Brzdek et al. (see [6, Theorem 1]).

First, we take the following three hypotheses (all notations come from [6]).

(H1) $U$ is a nonempty set, $V$ is a Banach space, $f_{1}, \ldots f_{k}: U \rightarrow U$ and $L_{1}, \ldots L_{k}: U \rightarrow \mathbb{R}_{+}$are given.

(H2) $\mathcal{T}: V^{U} \rightarrow V^{U}$ is an operator satisfying the inequality

$$
\|\mathcal{T} \xi(x)-\mathcal{T} \mu(x)\| \leq \sum_{i=1}^{k} L_{i}(x)\left\|\xi\left(f_{i}(x)\right)-\mu\left(f_{i}(x)\right)\right\|
$$

for all $\xi, \mu \in V^{U}, x \in U$.

(H3) $\Lambda: \mathbb{R}_{+}^{U} \rightarrow \mathbb{R}_{+}^{U}$ is a linear operator defined by

$$
\Lambda \delta(x):=\sum_{i=1}^{k} L_{i}(x) \delta\left(f_{i}(x)\right)
$$


for all $\delta \in \mathbb{R}_{+}^{U}, x \in U$.

The mentioned fixed point theorem is stated as follows.

Theorem 1.2. Let hypotheses $(\boldsymbol{H} 1)-(\boldsymbol{H} 3)$ be valid and functions $\varepsilon: U \rightarrow$ $\mathbb{R}_{+}$and let $\varphi: U \rightarrow V$ fulfil the following two conditions:

$$
\begin{gathered}
\|\mathcal{T} \varphi(x)-\varphi(x)\| \leq \varepsilon(x), \quad x \in U \\
\varepsilon^{*}(x):=\sum_{n=0}^{\infty} \Lambda^{n} \varepsilon(x)<\infty, \quad x \in U .
\end{gathered}
$$

Then, there exists a unique fixed point $\psi$ of $\mathcal{T}$ with

$$
\|\varphi(x)-\psi(x)\| \leq \varepsilon^{*}(x), \quad x \in U .
$$

Moreover

$$
\psi(x)=\lim _{n \rightarrow \infty} \mathcal{T}^{n} \varphi(x), \quad x \in U
$$

\section{Hyperstability Results of eq (1.3)}

The following theorems are the main results in this paper and concern the $\Sigma$-hyperstability of equation (1.3).

Theorem 2.1. Let $G$ be an 2-divisible abelian group and $E$ be a Banach space. If $f: G \rightarrow E$ satisfies

$$
\left\|2 f\left(\frac{x+y}{2}\right)+f\left(\frac{x-y}{2}\right)+f\left(\frac{y-x}{2}\right)-f(y)-f(x)\right\| \leq \varepsilon(x, y)
$$

for all $x, y \in G$ and $\varepsilon \in \Sigma$, then $f$ is a solution of (1.3) on $G$.

Proof. Let $\varepsilon \in \Sigma$, then there exists a constant $c \in \mathbb{R}_{+}$such that $\varepsilon(x, y)=$ $c H(\gamma(x), \gamma(y))$. Replacing $(x, y)$ by $(x, m x)$, with $m \in \mathbb{N}_{1}$, in $(2.1)$, we get

$$
\begin{aligned}
\| 2 f\left(\frac{1+m}{2} x\right) & +f\left(\frac{1-m}{2} x\right)+f\left(\frac{m-1}{2} x\right)-f(m x)-f(x) \| \\
& \leq \varepsilon(x, m x)=c H(\gamma(x), \gamma(m x)):=\varepsilon_{m}(x)
\end{aligned}
$$

for all $x \in G$. Further put

$\mathcal{T} \xi(x):=2 \xi\left(\frac{1+m}{2} x\right)+\xi\left(\frac{1-m}{2} x\right)+\xi\left(\frac{m-1}{2} x\right)-\xi(m x), \quad x \in G, \xi \in E^{G}$. 
Then the inequality (2.2) takes the form

$$
\|\mathcal{T} f(x)-f(x)\| \leq \varepsilon_{m}(x), \quad x \in G .
$$

Now, we define an operator $\Lambda: \mathbb{R}_{+}^{G} \rightarrow \mathbb{R}_{+}^{G}$ by

$$
\begin{aligned}
\Lambda \delta(x):= & 2 \delta\left(\frac{1+m}{2} x\right)+\delta\left(\frac{1-m}{2} x\right)+\delta\left(\frac{m-1}{2} x\right) \\
& +\delta(m x), x \in G, \quad \delta \in \mathbb{R}_{+}^{G} .
\end{aligned}
$$

This operator has the form described in (H3) with $k=4$ and $f_{1}(x)=\frac{1+m}{2} x$, $f_{2}(x)=\frac{1-m}{2} x, f_{3}(x)=\frac{m-1}{2} x, f_{4}(x)=m x, L_{1}(x)=2$ and $L_{2}(x)=L_{3}(x)=$ $L_{4}(x)=1$ for all $x \in G$.

Moreover, for every $\xi, \mu \in E^{G}$ and $x \in G$, we obtain

$$
\begin{aligned}
\|\mathcal{T} \xi(x)-\mathcal{T} \mu(x)\|= & \left\|2(\xi-\mu)\left(f_{1}(x)\right)+(\xi-\mu)\left(f_{2}(x)\right)(\xi-\mu)\left(f_{3}(x)\right)-(\xi-\mu)\left(f_{4}(x)\right)\right\| \\
\leq & 2\left\|(\xi-\mu)\left(f_{1}(x)\right)\right\|+\left\|(\xi-\mu)\left(f_{2}(x)\right)\right\|+\left\|(\xi-\mu)\left(f_{3}(x)\right)\right\| \\
& +\left\|(\xi-\mu)\left(f_{4}(x)\right)\right\| \\
& =\sum_{i=1}^{4} L_{i}(x)\left\|(\xi-\mu)\left(f_{i}(x)\right)\right\| .
\end{aligned}
$$

So, (H2) is valid. Not that for some $p<0$, we have

$$
\lim _{n \rightarrow \infty}\left(2\left(\frac{1+m}{2}\right)^{p}+2\left(\frac{m-1}{2}\right)^{p}+m^{p}\right)=0,
$$

then, there exists $m_{0} \in \mathbb{N}^{*}$ such that

$$
A_{m}:=2\left(\frac{1+m}{2}\right)^{p}+2\left(\frac{m-1}{2}\right)^{p}+m^{p}<1, \quad \text { for all } m \geq m_{0} .
$$

Therefore, in view of (1.7) and (2.3), it is easily to check that

$$
\begin{aligned}
\Lambda \varepsilon_{m}(x)= & 2 \varepsilon_{m}\left(\frac{1+m}{2} x\right)+\varepsilon_{m}\left(\frac{1-m}{2} x\right)+\varepsilon_{m}\left(\frac{m-1}{2} x\right)+\varepsilon_{m}(m x) \\
= & 2 H\left(\gamma\left(\frac{1+m}{2} x\right), \gamma\left(m \frac{1+m}{2} x\right)\right)+H\left(\gamma\left(\frac{1-m}{2} x\right), \gamma\left(m \frac{1-m}{2} x\right)\right) \\
& +H\left(\gamma\left(\frac{m-1}{2} x\right), \gamma\left(m \frac{m-1}{2} x\right)\right)+H(\gamma(m x), \gamma(m . m x)) \\
= & 2 H\left(\frac{1+m}{2} \gamma(x), \frac{1+m}{2} \gamma(m x)\right)+2 H\left(\frac{m-1}{2} \gamma(x), \frac{m-1}{2} \gamma(m x)\right) \\
& +H(m \gamma(x), m \gamma(m x)) \\
= & \left(2\left(\frac{1+m}{2}\right)^{p}+2\left(\frac{m-1}{2}\right)^{p}+m^{p}\right) H(\gamma(x), \gamma(m x)) \\
= & A_{m} \varepsilon_{m}(x)
\end{aligned}
$$


for all $x \in G$ and $m \geq m_{0}$. Therefore, we obtain that

$$
\begin{aligned}
\varepsilon_{m}^{*}(x): & =\sum_{n=0}^{\infty} \Lambda^{n} \varepsilon_{m}(x) \\
& =\varepsilon_{m}(x) \sum_{n=0}^{\infty}\left(A_{m}\right)^{n} \\
& =\frac{\varepsilon_{m}(x)}{1-A_{m}}<\infty .
\end{aligned}
$$

for all $x \in G$ and $m \geq m_{0}$. Thus, according to Theorem 1.2, for each $m \geq m_{0}$ there exists a unique solution $F_{m}: G \rightarrow E$ of the equation

$$
F_{m}(x)=2 F_{m}\left(\frac{1+m}{2} x\right)+F_{m}\left(\frac{1-m}{2} x\right)+F_{m}\left(\frac{m-1}{2} x\right)-F_{m}(m x)
$$

for all $x \in G$, such that

$$
\left\|f(x)-F_{m}(x)\right\| \leq \frac{\varepsilon_{m}(x)}{1-A_{m}}, \quad x \in G .
$$

Moreover $F_{m}(x)=\lim _{n \rightarrow \infty} \mathcal{T}^{n} f(x)$ for all $x \in G$.

To prove that the function $F_{m}$ satisfies the functional equation (1.3) on $G$, it suffices to prove the following inequality

$$
\left\|2 \mathcal{T}^{n} f\left(\frac{x+y}{2}\right)+\mathcal{T}^{n} f\left(\frac{x-y}{2}\right)+\mathcal{T}^{n} f\left(\frac{y-x}{2}\right)-\mathfrak{T}^{n} f(y)-\mathcal{T}^{n} f(x)\right\| \leq\left(A_{m}\right)^{n} \varepsilon(x, y)
$$

for all $x, y \in G$ and $n \in \mathbb{N}$. Indeed, if $n=0$, then (2.5) is simply (2.1). So, fix $n \in \mathbb{N}$ and suppose that (2.5) holds for $n$. Then

$$
\begin{aligned}
& \left\|2 \mathcal{T}^{n+1} f\left(\frac{x+y}{2}\right)+\mathcal{T}^{n+1} f\left(\frac{x-y}{2}\right)+\mathcal{T}^{n+1} f\left(\frac{y-x}{2}\right)-\mathcal{T}^{n+1} f(y)-\mathcal{T}^{n+1} f(x)\right\| \\
= & \| 2\left[2 \mathcal{T}^{n} f\left(\frac{m+1}{2} \frac{x+y}{2}\right)+\mathcal{T}^{n} f\left(\frac{1-m}{2} \frac{x+y}{2}\right)+\mathcal{T}^{n} f\left(\frac{m-1}{2} \frac{x+y}{2}\right)\right. \\
& \left.-\mathcal{T}^{n} f\left(m \frac{x+y}{2}\right)\right]+\left[2 \mathcal{T}^{n} f\left(\frac{m+1}{2} \frac{x-y}{2}\right)+\mathcal{T}^{n} f\left(\frac{1-m}{2} \frac{x-y}{2}\right)\right. \\
& \left.+\mathcal{T}^{n} f\left(\frac{m-1}{2} \frac{x-y}{2}\right)-\mathcal{T}^{n} f\left(m \frac{x-y}{2}\right)\right]+\left[2 \mathcal{T}^{n} f\left(\frac{m+1}{2} \frac{y-x}{2}\right)\right. \\
& \left.+\mathcal{T}^{n} f\left(\frac{1-m}{2} \frac{y-x}{2}\right)+\mathcal{T}^{n} f\left(\frac{m-1}{2} \frac{y-x}{2}\right)-\mathcal{T}^{n} f\left(m \frac{y-x}{2}\right)\right] \\
& -\left[2 \mathcal{T}^{n} f\left(\frac{m+1}{2} y\right)+\mathcal{T}^{n} f\left(\frac{1-m}{2} y\right)+\mathcal{T}^{n} f\left(\frac{m-1}{2} y\right)-\mathcal{T}^{n} f(m y)\right]
\end{aligned}
$$




$$
\begin{aligned}
& -\left[2 \mathcal{T}^{n} f\left(\frac{m+1}{2} x\right)+\mathcal{T}^{n} f\left(\frac{1-m}{2} x\right)+\mathcal{T}^{n} f\left(\frac{m-1}{2} x\right)-\mathcal{T}^{n} f(m x)\right] \| \\
\leq & 2 \| 2 \mathcal{T}^{n} f\left(\frac{m+1}{2} \frac{x+y}{2}\right)+\mathcal{T}^{n} f\left(\frac{m+1}{2} \frac{x-y}{2}\right)+\mathcal{T}^{n} f\left(\frac{m+1}{2} \frac{y-x}{2}\right) \\
& -\mathcal{T}^{n} f\left(\frac{m+1}{2} y\right)-\mathcal{T}^{n} f\left(\frac{m+1}{2} x\right)\|+\| 2 \mathcal{T}^{n} f\left(\frac{1-m}{2} \frac{x+y}{2}\right) \\
& +\mathcal{T}^{n} f\left(\frac{1-m}{2} \frac{x-y}{2}\right)+\mathcal{T}^{n} f\left(\frac{1-m}{2} \frac{y-x}{2}\right)-\mathcal{T}^{n} f\left(\frac{1-m}{2} y\right) \\
& -\mathcal{T}^{n} f\left(\frac{1-m}{2} x\right)\|+\| 2 \mathcal{T}^{n} f\left(\frac{m-1}{2} \frac{x+y}{2}\right)+\mathfrak{T}^{n} f\left(\frac{m-1}{2} \frac{x-y}{2}\right) \\
& +\mathcal{T}^{n} f\left(\frac{m-1}{2} \frac{y-x}{2}\right)-\mathcal{T}^{n} f\left(\frac{m-1}{2} y\right)-\mathcal{T}^{n} f\left(\frac{m-1}{2} x\right) \| \\
& +\left\|2 \mathcal{T}^{n} f\left(m \frac{x+y}{2}\right)+\mathcal{T}^{n} f\left(m \frac{x-y}{2}\right)+\mathcal{T}^{n} f\left(m \frac{y-x}{2}\right)-\mathcal{T}^{n} f(m y)-\mathcal{T}^{n} f(m x)\right\| \\
\leq & \left(A_{m}\right)^{n}\left[2 \varepsilon\left(\frac{m+1}{2} x, \frac{m+1}{2} y\right)+\varepsilon\left(\frac{1-m}{2} x, \frac{1-m}{2} y\right)+\varepsilon\left(\frac{m-1}{2} x, \frac{m-1}{2} y\right)\right. \\
& \quad+\varepsilon(m x, m y)] \\
= & \left(A_{m}\right)^{n+1} \varepsilon(x, y)
\end{aligned}
$$

for all $x, y \in G$. Thus, by induction, we have shown that (2.5) holds for all $x, y \in G$ and for all $n \in \mathbb{N}$. Letting $n \rightarrow \infty$ in (2.5), we obtain

$$
2 F_{m}\left(\frac{x+y}{2}\right)+F_{m}\left(\frac{x-y}{2}\right)+F_{m}\left(\frac{y-x}{2}\right)=F_{m}(y)+F_{m}(x)
$$

for all $x, y \in G$. So, we find a sequence $\left(F_{m}\right)_{m \geq m_{0}}$ satisfies (1.3) on $G$ such that

$$
\left\|f(x)-F_{m}(x)\right\| \leq \frac{\varepsilon_{m}(x)}{1-A_{m}}, \quad x \in G, \quad m \geq m_{0} .
$$

Next, we prove that $F_{m}=F_{k}$ for all $m, k \in \mathbb{N}_{m_{0}}$. Let us fix $m, k \in \mathbb{N}_{m_{0}}$ and note that $F_{m}$ and $F_{k}$ satisfy $(2.6)$. Hence, by replacing $(x, y)$ by $(x, m x)$ in (2.6), we get $\mathcal{T} F_{m}(x)=F_{m}(x), \mathcal{T} F_{k}(x)=F_{k}(x)$ for all $x \in G$ and

$$
\left\|F_{m}(x)-F_{k}(x)\right\| \leq \frac{\varepsilon_{m}(x)}{1-A_{m}}+\frac{\varepsilon_{k}(x)}{1-A_{k}}
$$


for all $x \in G$. It follows, by linearity of $\Lambda$ and (2.4) that

$$
\begin{aligned}
\left\|F_{m}(x)-F_{k}(x)\right\| & =\left\|\mathcal{T}^{n} F_{m}(x)-\mathcal{T}^{n} F_{k}(x)\right\| \\
& \leq \frac{\Lambda^{n} \varepsilon_{m}(x)}{1-A_{m}}+\frac{\Lambda^{n} \varepsilon_{k}(x)}{1-A_{k}} \\
& \leq\left(A_{m}\right)^{n}\left[\frac{\varepsilon_{m}(x)}{1-A_{m}}+\frac{\varepsilon_{k}(x)}{1-A_{k}}\right]
\end{aligned}
$$

for all $x \in G$ and $n \in \mathbb{N}$. Letting $n \rightarrow \infty$ we get $F_{m}=F_{k}=: F$. Thus, we have

$$
\|f(x)-F(x)\| \leq \frac{\varepsilon_{m}(x)}{1-A_{m}}, \quad x \in G, \quad m \geq m_{0}
$$

and the function $F$ is a solution of (1.3).

To prove the uniqueness of the function $F$, let us assume that there exists a function $F^{\prime}: G \rightarrow E$ which satisfies (1.3) and the inequality

$$
\left\|f(x)-F^{\prime}(x)\right\| \leq \frac{\varepsilon_{m}(x)}{1-A_{m}}, \quad x \in G, \quad m \geq m_{0} .
$$

Then

$$
\left\|F(x)-F^{\prime}(x)\right\| \leq \frac{2 \varepsilon_{m}(x)}{1-A_{m}}, \quad x \in G, \quad m \geq m_{0} .
$$

Further $\mathcal{T} F^{\prime}(x)=F^{\prime}(x)$ for all $x \in G$. Therefore, with a fixed $m \in \mathbb{N}_{m_{0}}$

$$
\begin{aligned}
\left\|F(x)-F^{\prime}(x)\right\| & =\left\|\mathcal{T}^{n} F(x)-\mathcal{T}^{n} F^{\prime}(x)\right\| \\
& \leq \frac{2 \Lambda^{n} \varepsilon_{m}(x)}{1-A_{m}} \\
& \leq 2\left(A_{m}\right)^{n} \times \frac{\varepsilon_{m}(x)}{1-A_{m}}
\end{aligned}
$$

for all $x \in G$ and $n \in \mathbb{N}$. Letting $n \rightarrow \infty$ we get $F=F^{\prime}$, which yields

$$
\|f(x)-F(x)\| \leq \frac{\varepsilon_{m}(x)}{1-A_{m}}, \quad x \in G, \quad m \geq m_{0} .
$$

Next, in view of (1.6), we have

$$
\inf \left\{\frac{\varepsilon_{m}(x)}{1-A_{m}}: m \geq m_{0}\right\}=0
$$

for all $x \in G$, this means that $f(x)=F(x)$ for $x \in G$, which implies that $f$ satisfies the functional equation (1.3) on $G$ and the proof of the theorem is complete. 
In a similar way we can prove that Theorem (2.1) holds if the inequality (2.1) is defined on $G \backslash\{0\}:=G_{0}$.

Theorem 2.2. Let $G$ be an 2-divisible abelian group and $E$ be a Banach space. Let $\Sigma$ be the set of all functions $\varepsilon: G_{0} \rightarrow \mathbb{R}_{+}$which satisfy the conditions as stated in the Section (1). If $f: G \rightarrow E$ satisfies

$$
\left\|2 f\left(\frac{x+y}{2}\right)+f\left(\frac{x-y}{2}\right)+f\left(\frac{y-x}{2}\right)-f(y)-f(x)\right\| \leq \varepsilon(x, y)
$$

for all $x, y \in G_{0}$ and $\varepsilon \in \Sigma$, then $f$ is a solution of (1.3) on $G_{0}$.

\section{Applications}

In this section we give some applications of the Theorem 2.2, with the case:

$$
\varepsilon(x, y)=\theta\|x\|^{p} \cdot\|y\|^{q}
$$

where $\theta \in \mathbb{R}_{+}, p, q \in \mathbb{R}$ and $x, y \neq 0$.

Corollary 3.1. Let $E_{1}$ and $E_{2}$ ba a normed space and a Banach space, respectively. Assume $S:=(S,+)$ is an 2-divisible subgroup of the group $\left(E_{1},+\right), p, q \in \mathbb{R}, p+q<0$ and $\theta \geq 0$. If $f: S \rightarrow E_{2}$ satisfies

$$
\left\|2 f\left(\frac{x+y}{2}\right)+f\left(\frac{x-y}{2}\right)+f\left(\frac{y-x}{2}\right)-f(y)-f(x)\right\| \leq \theta\|x\|^{p}\|y\|^{q}
$$

for all $x, y \in S \backslash\{0\}$, then $f$ is a solution of (1.3) on $S \backslash\{0\}$.

Proof. Let $\Sigma$ the set of all functions $\varepsilon: S \backslash\{0\} \times S \backslash\{0\} \rightarrow \mathbb{R}_{+}$such that

$$
\varepsilon(x, y)=\theta\|x\|^{p}\|y\|^{q}
$$

for some $\theta \in \mathbb{R}_{+}$and for all $x, y \in S \backslash\{0\}$. Define $H: \mathbb{R}_{+*}^{2} \rightarrow \mathbb{R}_{+}$by $H(u, v)=c u^{p} v^{q}$ for some $c \geq 0, p+q<0$ and for all $u, v \in \mathbb{R}_{+*}$ and $\gamma: S \rightarrow \mathbb{R}_{+}$by $\gamma(x)=\|x\|$ for all $x \in S$. It is easily seen that $H$ is monotonically symmetric homogeneous function of degree $p+q<0$ and conditions indicated in the start of the second section are fulfilled. Therefore every function $f: S \backslash\{0\} \rightarrow E_{2}$ satisfying (3.1) is a solution of the functional equation (1.3) on $S \backslash\{0\}$. 
We know that any norm that satisfies the parallelogram law is bound to have been originated from a scalar product. The following corollary gives a characterization of the inner product space, which is one of the applications of Corollary 3.1.

Corollary 3.2. Let $X$ be a normed space and $X_{0}=X \backslash\{0\}$. Write

$$
D(x, y)=\left|2\left\|\frac{x+y}{2}\right\|^{2}+\left\|\frac{x-y}{2}\right\|^{2}+\left\|\frac{y-x}{2}\right\|^{2}-\|y\|^{2}-\|x\|^{2}\right|
$$

for all $x, y \in X$. Assume that

$$
\sup _{x, y \in X_{0}} \frac{D(x, y)}{\|x\|^{p}\|y\|^{q}}<\infty
$$

for somme $p, q \in \mathbb{R}$ and $p+q<0$. Then $X$ is an inner product space.

Proof. Write $f(x)=\|x\|^{2}$. Then from Corollary 3.1, we easily derive $f$ is a solution of the functional equation (1.3). That implies $D(x, y)=0$. Thus, the norm $\|$.$\| on X$ satisfies the parallelogram law:

$$
\|x+y\|^{2}+\|x-y\|^{2}=2\|x\|^{2}+2\|y\|^{2}, \quad x, y \in X .
$$

Therefore, $X$ is an inner product space.

Corollary 3.3. Let $G$ be an 2-divisible commutative group and $E$ be a $B a$ nach space. Let $\Sigma$ be the set of all functions $\varepsilon: G \rightarrow \mathbb{R}_{+}$which satisfy the conditions as stated in the Section (1) and $F: G^{2} \rightarrow E$ be a mapping such that $F\left(x_{0}, y_{0}\right) \neq 0$ for some $x_{0}, y_{0} \in G$ and

$$
\|F(x, y)\| \leq \varepsilon(x, y)
$$

for all $x, y \in G$. Then the functional equation

$2 h\left(\frac{x+y}{2}\right)+h\left(\frac{x-y}{2}\right)+h\left(\frac{y-x}{2}\right)=F(x, y)+h(y)+h(x), \quad x, y, z \in G$

has no solution in the class of functions $h: G \rightarrow E$.

Proof. Suppose that $h: G \rightarrow E$ is a solution to (3.3). Then (2.1) holds, and consequently, according to the above theorems, $h$ is Jensen on $G$, which means that $F\left(x_{0}, y_{0}\right)=0$. This is a contradiction. 


\section{Acknowledgement}

The author would like to thanks the anonymous referee for his careful reading and valuable suggestions to improve the quality of the paper.

\section{References}

[1] M. A. Abolfathi, A. Ebadian, and R. Aghalary, Stability of mixed additivequadratic Jensen type functional equation in non-Archimedean $\ell$-fuzzy normed spaces, Annali dell'Universit di Ferrara, 60, (2014), 307-319

[2] T. Aoki, On the stability of the linear transformation in Banach spaces, J. Math. Soc. Japan, 2, (1950), 64-66

[3] C. Baak, Cauchy-Rassias stability of Cauchy-Jensen additive mappings in Banach spaces, Acta Math. Sin. (Engl. Ser.), 22, (2006), 1789-1796

[4] A. Bahyrycz and M. Piszczek, Hyperstability of the Jensen functional equation, Acta Math. Hungar., 142, (2014), 353-365

[5] D. G. Bourgin, Approximately isometric and multiplicative transformations on continuous function rings, Duke Math. J., 16, (1949), 385-397

[6] J. Brzdek, J. Chudziak, and Z. Páles, A fixed point approach to stability of functional equations, Nonlinear Anal., 74, (2011), 6728-6732

[7] J. Brzdek, Remarks on hyperstability of the the Cauchy equation, Aequations Mathematicae, 86, (2013), 255-267

[8] J. Brzdek, Hyperstability of the Cauchy equation on restricted domains, Acta Math. Hungarica, 141, (2013), 58-67

[9] J. Brzdek and K. Ciepliński, Hyperstability and superstability, Abstract and Applied Analysis. Special Issue., (2013), 1-13

[10] J. Brzdek, A hyperstability result for the Cauchy equation, Bulletin of the Australian Mathematical Society, 89, (2014), 33-40

[11] L. Cădariu and V. Radu, Fixed points and the stability of Jensens functional equation, Journal of Inequalities in Pure and Applied Mathematics, 4, (2003)

[12] Iz. EL-Fassi and S. Kabbaj, On the hyperstability of a Cauchy-Jensen type functional equation in Banach spaces, Proyecciones J. Math., 34, (2015), 359-375

[13] P. Găvruta, A generalization of the Hyers-Ulam-Rassias stability of approximately additive mappings, J Math. Anal. Appl., 184, (1994), 431-436

[14] M. E. Gordji, H. Khodaei, and M. Kamyar, Stability of Cauchy-Jensen type functional equation in generalized fuzzy normed spaces, Computers and Mathematics with Applications, 62, (2011), 2950- 2960

[15] E. Gselmann, Hyperstability of a functional equation, Acta Mathematica Hungarica, 124, (2009), 179-188 
Vol. LIII (2015) The hyperstability of a mixed additive-quadratic-Jensen... 71

[16] D. H. Hyers, On the stability of the linear functional equation, Proc. Nat. Acad. Sci., U.S.A., 27, (1941), 222-224

[17] K. W. Jun, H. M. Kim, and J. M. Rassias, Extended Hyers-Ulam stability for Cauchy-Jensen mappings, J. Diference Equ. Appl., 13, (2007), 1139-1153

[18] S.-M. Jung, M. S. Moslehian, and P. K. Sahoo, Stability of a generalized Jensen equation on restricted domains, J. Math. Ineq., 4, (2010), 191-206

[19] S.-M. Jung, D.Popa, and M. Th. Rassias, On the stability of the linear functional equation in a single variable on complete metric groups, Journal of Global Optimization, 59, (2014), 165-171

[20] Z. Kominek, On a local stability of the Jensen functional equation, Demonstratio Math., 22, (1989), 499-507

[21] Y.-H. Lee and K. W. Jun, A generalization of the Hyers-Ulam-Rassias stability of Jensens equation, J. Math. Anal. Appl., 238, (1999), 305-315

[22] Y.-H. Lee, S.- M. Jung, and M. Th. Rassias, On an n-dimensional mixed type additive and quadratic functional functional equation, Applied Mathematics and Computation, 228, (2014), 13 -16

[23] G. Maksa and Z. Páles, Hyperstability of a class of linear functional equations, Acta Math., 17, (2001), 107-112

[24] C. Park, Fixed points and Hyers-Ulam-Rassias stability of Cauchy-Jensen functional equations in Banach algebras, Fixed. Point. Theory. Appl., 15, (2007)

[25] C.G. Park and Th.M. Rassias, Hyers -Ulam stability of a generalized Apollonius type quadratic mapping, Journal of Mathematical Analysis and Applications, 322, 371-381

[26] M. Piszczek, Remark on hyperstability of the general linear equation, Aequations Mathematicae, 88, (2013), 163-168

[27] Th. M. Rassias, On the stability of the linear mapping in Banach spaces, Proc. Amer. Math. Soc., 72, (1978), 297-300

[28] J. M. Rassias, On the Ulam stability of Jensen and Jensen type mappings on restricted domains, J. Math. Anal. Appl., 281, (2003), 516-524

[29] S. M. Ulam, Problems in Modern Mathematics. Chapter IV, Science Editions, Wiley, New York, 1960

[30] X. Yang, L. Chang, and G. Liu, Orthogonal Stability of Mixed Additive-Quadratic Jensen Type Functional Equation in Multi-Banach Spaces, Advances in Pure Mathematics, 5, (2015), 325-332

Iz-iddine EL-Fassi*

Department of Mathematics,

Faculty of Sciences,

University of Ibn Tofail,

Kenitra, Morocco. 
E-mail: izidd-math@hotmail.fr

Samir Kabbaj

Department of Mathematics,

Faculty of Sciences,

University of Ibn Tofail,

Kenitra, Morocco.

E-mail: samkabbaj@yahoo.fr

Received: 29.08.2015

Accepted: 15.01.2016 